INPLASY

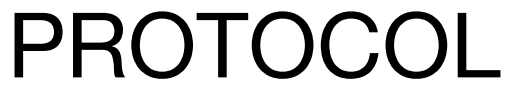

To cite: Feng et al. Effects of Pseudomonas aeruginosa infection on the prognosis of patients with chronic obstructive pulmonary disease: a systematic review and meta-analysis. Inplasy protocol 202140092. doi: 10.37766/inplasy2021.4.0092

Received: 17 April 2021

Published: 18 April 2021

Corresponding author: XinYu Feng

775287759@qq.com

Author Affiliation: Liyuan hospital,Tongji Medical College, Huazhong University of Science \& Technology

Support: YuLan Zeng funded this study.

Review Stage at time of this submission: Piloting of the study selection process.

Conflicts of interest:

None declared.

\section{Effects of Pseudomonas aeruginosa infection on the prognosis of patients with chronic obstructive pulmonary disease: a systematic review and meta-analysis}

Feng, $X Y^{1}$; Zeng, $Y L^{2}$.

Review question / Objective: The objective of this systematic review and meta-analysis is to evaluate whether Pseudomonas aeruginosa infection has an impact on the prognosis of patients with COPD. Outcome measures included mortality, readmission rate, length of stay, lung function, and BODE index.

Condition being studied: Chronic obstructive pulmonary disease (COPD) is a disease characterized by persistent progressive airflow limitation and is now the third leading cause of death worldwide. The increased morbidity and mortality of COPD imposes a significant medical and economic burden on the world. The adverse outcomes of COPD are often associated with respiratory infections.

INPLASY registration number: This protocol was registered with the International Platform of Registered Systematic Review and Meta-Analysis Protocols (INPLASY) on 18 April 2021 and was last updated on 18 April 2021 (registration number INPLASY202140092).

\section{INTRODUCTION}

Review question / Objective: The objective of this systematic review and meta-analysis is to evaluate whether Pseudomonas aeruginosa infection has an impact on the prognosis of patients with COPD. Outcome measures included mortality, readmission rate, length of stay, lung function, and BODE index.

Condition being studied: Chronic obstructive pulmonary disease (COPD) is a disease characterized by persistent 
progressive airflow limitation and is now the third leading cause of death worldwide. The increased morbidity and mortality of COPD imposes a significant medical and economic burden on the world. The adverse outcomes of COPD are often associated with respiratory infections.

\section{METHODS}

Participant or population: Adults with COPD(as diagnosed by Global Initiative for the Diagnosis, Management, and Prevention of Chronic Obstructive Lung Disease) with or without Pseudomonas aeruginoa infection will be include, regardless of gender, age, ethnicity, disease duration, severity.

Intervention: Exposure was defined as any Pseudomonas aeruginoa positive culture samples obtained from the lower respiratory tract (sputum, trachea secretions, bronchial secretions, and bronchial alveolar lavage) during the hospitalization of the study participant.

Comparator: Adults with COPD(as diagnosed by Global Initiative for the Diagnosis, Management, and Prevention of Chronic Obstructive Lung Disease)without Pseudomonas aeruginoa infection.

Study designs to be included: Cohort studies, randomized controlled trials or cross-sectional studies will be included.

Eligibility criteria: 1- Studies evaluating the effect of Pseudomonas aeruginosa infection on mortality in patients with COPD 2- Studies evaluating other outcomes such as readmission rate, length of hospital stay, lung function, and BODE index in patients with and without COPD with and without Pseudomonas aeruginosa infection. 3- Randomized controlled trials, prospective observational trials with control groups or retrospective trials. 4Studies published in peer-reviewed scientific journals, conference reports. 5Studies published in the English language.
Information sources: We will search, with no time restrictions, the following databases for relevant English language literature: PubMed, Embase, MEDLINE, the Cochrane library and Web of Science.The search string will be built as follows: (COPD OR Chronic Obstructive Pulmonary Disease OR Chronic airflow obstruction OR chronic obstructive airflow disease OR chronic obstructive lung disease) AND (Pseudomonas aeruginosa) AND (morbidity OR mortality OR survival OR prognosis OR duration of hospital stay OR quality of life OR readmission rate). The electronic database search will be supplemented by a manual search of the reference lists of included articles.

Main outcome(s): Mortality, Readmission rate, Length of Stay(LOS), Forced expiratory volume in one second(FEV1), BODE index.

Quality assessment / Risk of bias analysis: The Newcastle-Ottawa Quality Assessment Scale (NOS) was used to assess the risk of bias in the studies included by the two authors (Z.Y.L and F.X.Y).NOS consisted of 8 items in 3 domains: selection (Representativeness of exposure cohort, Representativeness of non-exposed cohort, Ascertainment of exposure, Demonstration that outcome of interest was not present at start of study), comparability (Comparability of cohort on the basis of the design and analysis), and outcome/exposure (Assessment of Outcome, Adequate follow-up, Integrity of follow-up)for cohort studies.Similarly, disagreements were addressed through consensus.

Strategy of data synthesis: Risk ratio(RR) for both fixed and random effects models(weighting by inverse of variance) will be used. A continuity correction will also be used for cells with zero values. Between-study heterogeneity will be assessed using the $\mathrm{r} 2, \mathrm{X2}$ (Cochran $\mathrm{Q}$ ) and Pstatistics. According to the Cochrane handbook,the 2 will be considered nonimportant(60\%). Results will be assessed using forest plots and presented as RRs for 
the main outcome and secondary outcomes. An influence analysis will be performed to ascertain the results of the meta-analysis by excluding each of the individual studies. Publication bias will be assessed by a funnel plot for meta-analysis and quantified by the Egger and begg method. Statistical analysis will be conducted using STATA/MP 16.0.

Subgroup analysis: We will consider subgroups such as cohort type, region, sample size and year.

Sensitivity analysis: When heterogeneity was present, sensitivity analysis was performed by STATA 16.0 software to eliminate heterogeneity by removing each individual study one by one or the studies with poor quality.

Language: Chinese.

Country(ies) involved: China.

Keywords: Chronic obstructive pulmonary disease; Pseudomonas aeruginosa; Prognosis; Meta-analysis; Systematic review.

Contributions of each author:

Author 1 - XinYu Feng.

Email: 775287759@qq.com

Author 2 - YuLan Zeng.

Email: 1989LY0551@hust.edu.Cn 